\title{
The Correct Regularity Condition and Interpretation of Asymmetry in EGARCH
}

\author{
C.-L. Chang a $\underline{\text { M. McAleer }}^{\text {b,c,d,e,f a }}$ \\ ${ }^{a}$ Department of Applied Economics and Department of Finance,National Chung Hsing University, Taiwan, \\ ${ }^{b}$ Department of Quantitative Finance, National Tsing Hua University, Taiwan \\ ${ }^{c}$ Discipline of Business Analytics, University of Sydney Business School, Australia \\ ${ }^{d}$ Erasmus School of Economics, Erasmus University Rotterdam, The Netherlands, \\ ${ }^{e}$ Department of Quantitative Economics, Complutense University of Madrid, Spain \\ ${ }^{f}$ Institute of Advanced Sciences, Yokohama National University, Japan \\ Email: michael.mcaleer@gmail.com
}

\begin{abstract}
In the class of univariate conditional volatility models, the three most popular are the generalized autoregressive conditional heteroskedasticity (GARCH) model of Engle (1982) and Bollerslev (1986), the GJR (or threshold GARCH) model of Glosten, Jagannathan and Runkle (1992), and the exponential GARCH (or EGARCH) model of Nelson (1990, 1991). For purposes of deriving the mathematical regularity properties, including invertibility, to determine the likelihood function for estimation, and the statistical conditions to establish asymptotic properties, it is convenient to understand the stochastic properties underlying the three univariate models. The random coefficient autoregressive process was used to obtain GARCH by Tsay (1987), an extension of which was used by McAleer (2014) to obtain GJR. A random coefficient complex nonlinear moving average process was used by McAleer and Hafner (2014) to obtain EGARCH. These models can be used to capture asymmetry, which denotes the different effects on conditional volatility of positive and negative effects of equal magnitude, and possibly also leverage, which is the negative correlation between returns shocks and subsequent shocks to volatility (see Black 1976F). McAleer (2014) showed that asymmetry was possible for GJR, but not leverage. McAleer and Hafner (2014) showed that leverage was not possible for EGARCH. Surprisingly, the conditions for asymmetry in EGARCH seem to have been ignored in the literature, or have concentrated on the incorrect conditions, with no clear explanation, and hence with associated misleading interpretations. The purpose of the paper is to derive the regularity condition for asymmetry in EGARCH to provide the correct interpretation. It is shown that, in practice, EGARCH always displays asymmetry, though not leverage.
\end{abstract}

Keywords: Conditional volatility models, random coefficient complex nonlinear moving average process, EGARCH, asymmetry, leverage, regularity condition 


\section{INTRODUCTION}

In the class of univariate conditional volatility models, the three most popular are the generalized autoregressive conditional heteroskedasticity (GARCH) model of Engle (1982) and Bollerslev (1986), the GJR (or threshold GARCH) model of Glosten, Jagannathan and Runkle (1992), and the exponential GARCH (or EGARCH) model of Nelson $(1990,1991)$. Each of these models is widely read, used in practice, and highly cited.

For purposes of deriving the mathematical regularity properties, including invertibility, to determine the likelihood function for estimation, and the statistical conditions to establish asymptotic properties, it is convenient to understand the stochastic properties underlying the three univariate models. The random coefficient autoregressive process was used to obtain GARCH by Tsay (1987), an extension of which was used by McAleer (2004) to obtain GJR. A random coefficient complex nonlinear moving average process was used by McAleer and Hafner (2014) to obtain EGARCH.

These models can be used to capture asymmetry, which denotes the different impacts on conditional volatility of positive and negative shocks of equal magnitude, and possibly also leverage, which is the negative correlation between returns shocks and subsequent shocks to volatility (see Black 1979). McAleer (2014) showed that asymmetry was possible for GJR, but not leverage. McAleer and Hafner showed that leverage was not possible for EGARCH.

Surprisingly, the condition for asymmetry in EGARCH seems to have been ignored in the literature, or has concentrated on an incorrect condition, with no clear explanation, and hence with associated misleading interpretations. The purpose of the paper is to derive the regularity condition for asymmetry in EGARCH to provide the correct interpretation. It is shown that, in practice, EGARCH always displays asymmetry, though not leverage.

The paper is organized as follows. In Section 2, the GARCH and EGARCH models are derived from different underlying stochastic processes that lead to their derivation, the first from a random coefficient autoregressive process, and the second from a random coefficient complex nonlinear moving average process. Asymmetry and leverage are discussed in Section 3. The correct regularity condition for asymmetry in EGARCH is derived, and the correct interpretation is given, in Section 4. Some concluding comments are presented in Section 5.

\section{STOCHASTIC PROCESSES FOR GARCH AND EGARCH}

\subsection{Random Coefficient Autoregressive Process - GARCH}

Consider the conditional mean of financial returns, as follows:

$$
y_{t}=E\left(y_{t} \mid I_{t-1}\right)+\varepsilon_{t}
$$

where the financial returns, $y_{t}=\Delta \log P_{t}$, represent the log-difference in financial commodity prices, $P_{t}, I_{t-1}$ is the information set at time $t-1$, and $\varepsilon_{t}$ is a conditionally heteroskedastic error term, or returns shock. In order to derive conditional volatility specifications, it is necessary to specify the stochastic processes underlying the returns shocks, $\varepsilon_{t}$.

Now consider the random coefficient AR(1) process underlying the return shocks, $\varepsilon_{t}$ :

$$
\varepsilon_{t}=\phi_{t} \varepsilon_{t-1}+\eta_{t}
$$

where

$\phi_{t} \sim i i d(0, \alpha), \alpha \geq 0$,

$\eta_{t} \sim \operatorname{iid}(0, \omega), \omega \geq 0$,

$\eta_{t}=\varepsilon_{t} / \sqrt{h_{t}}$ is the standardized residual, with $h_{t}$ defined below. 
Tsay (1987) derived the $\mathrm{ARCH}(1)$ model of Engle (1982) from equation (2) as:

$$
h_{t} \equiv E\left(\varepsilon_{t}^{2} \mid I_{t-1}\right)=\omega+\alpha \varepsilon_{t-1}^{2}
$$

where $h_{t}$ represents conditional volatility, and $I_{t-1}$ is the information set available at time $t$ - 1 . A lagged dependent variable, $h_{t-1}$, is typically added to equation (3) to improve the sample fit:

$$
h_{t} \equiv E\left(\varepsilon_{t}^{2} \mid I_{t-1}\right)=\omega+\alpha \varepsilon_{t-1}^{2}+\beta h_{t-1}
$$

From the specification of equation (2), it is clear that both $\omega$ and $\alpha$ should be positive as they are the unconditional variances of two different stochastic processes. Moreover, as GARCH is symmetric, there is no asymmetry or leverage.

Given the non-normality of the returns shocks, the Quasi-Maximum Likelihood Estimators (QMLE) of the parameters have been shown to be consistent and asymptotically normal in several papers. For example, Ling and McAleer (2003) showed that the QMLE for a generalized $\operatorname{ARCH}(p, q)($ or $\operatorname{GARCH}(p, q))$ is consistent if the second moment is finite. A sufficient condition for the QMLE of $\operatorname{GARCH}(1,1)$ in equation (4) to be consistent and asymptotically normal is $\alpha+\beta<1$.

\subsection{Random Coefficient Complex Nonlinear Moving Average Process - EGARCH}

A conditional volatility model that can accommodate asymmetry is the EGARCH model of Nelson (1990, 1991). McAleer and Hafner (2014) showed that EGARCH, specifically EARCH(1) = EGARCH $(1,0)$, could be derived from a random coefficient complex nonlinear moving average (RCCNMA) process, as follows:

$$
\varepsilon_{t}=\phi_{t} \sqrt{\left|\eta_{t-1}\right|}+\psi_{t} \sqrt{\eta_{t-1}}+\eta_{t}
$$

where

$$
\begin{aligned}
& \phi_{t} \sim \operatorname{iid}(0, \alpha), \\
& \psi_{t} \sim \operatorname{iid}(0, \gamma), \\
& \eta_{t} \sim \operatorname{iid}(0, \omega),
\end{aligned}
$$

$\sqrt{\eta_{t-1}}$ is a complex-valued function of $\eta_{t-1}$,

and $\eta_{t}=\varepsilon_{t} / \sqrt{h_{t}}$ is the standardized residual.

McAleer and Hafner (2014) show that the conditional variance of the squared returns shocks in equation (5) is:

$$
h_{t}=E\left(\varepsilon_{t}^{2} \mid I_{t-1}\right)=\omega+\alpha\left|\eta_{t-1}\right|+\gamma \eta_{t-1}
$$

where it is clear from the RCCNMA process in equation (5) that all three parameters should be positive as they are the variances of three different stochastic processes. The constant $\omega$ is not equivalent to the unconditional variance of $\eta_{t}$. In (6), it is assumed that $h_{t}>0$ as $\eta_{t-1}$ could be negative, which is equivalent to assuming $\alpha>\gamma$. This is not a problem when the logarithmic approximation is used in (7). 
Although the transformation of $h_{t}$ in equation (6) is not logarithmic, the approximation given by:

$$
\log h_{t}=\log \left(1+\left(h_{t}-1\right)\right) \approx h_{t}-1
$$

can be used to replace $h_{t}$ in equation (6) with $1+\log h_{t}$ to lead to $\operatorname{EARCH}(1)=\operatorname{EGARCH}(1,0)$ :

$$
\log h_{t}=E\left(\varepsilon_{t}^{2} \mid I_{t-1}\right)=(\omega-1)+\alpha\left|\eta_{t-1}\right|+\gamma \eta_{t-1},
$$

The use of an infinite lag for the RCCNMA process in equation (5) would yield the standard EGARCH model with lagged conditional volatility, namely $\operatorname{EGARCH}(1,1)$ :

$$
\log h_{t}=E\left(\varepsilon_{t}^{2} \mid I_{t-1}\right)=(\omega-1)+\alpha\left|\eta_{t-1}\right|+\gamma \eta_{t-1}+\beta \log h_{t-1}
$$

As EGARCH can be derived from a random coefficient complex nonlinear moving average (RCCNMA) process, it follows from the specification in equation (5) that there is no invertibility condition to transform the returns shocks, $\mathcal{E}_{t}$, to the standardized residuals, $\eta_{t}$. Therefore, there are as yet no asymptotic properties of the QMLE of the parameters of EGARCH.

Recently, Martinet and McAleer (2018) showed that the $\operatorname{EGARCH}(p, q)$ model could be derived from an alternative stochastic process, for which the invertibility condition can be stated simply and explicitly. This theoretical result is likely to lead to the development of asymptotic properties for the QMLE of EGARCH.

\section{ASYMMETRY AND LEVERAGE FOR EGARCH(1,1)}

The regularity conditions for asymmetry and leverage in EGARCH have been discussed in several different and conflicting ways, as given below. The specific definition of asymmetry, in particular, depends on what is meant by volatility, namely $h_{t}, \log h_{t}$ or $\sqrt{h_{t}}$. If a specification is symmetric with one metric, it may not be in others, except around 0 , by the results given above, so that the definition is only around 0 unless the volatility concept is specified.

(1) McAleer and Hafner (2014) showed that leverage exists for $\operatorname{EARCH}(1,1)$ if:

Regularity condition for leverage: $\gamma<0$ and $\gamma<\alpha<-\gamma$.

It is clear that leverage is not possible for $\operatorname{EGARCH}(1,1)$ as both $\alpha$ and $\gamma$, which are the variances of two stochastic processes, must be positive. It is possible for $\alpha$ to be positive in the second parametric condition for leverage, but it is typically omitted in the literature on EGARCH, without explanation.

(2) Nelson (1990) did not mention asymmetry or leverage. In the development of EGARCH, specifically $\operatorname{EARCH}(1)=\operatorname{EGARCH}(1,0)$, in a subsequent paper Nelson (1991) gave a definition of asymmetry, but did not provide any regularity condition. In discussing leverage, Nelson (1991, p. 351) considers two sets of conditions [using the terminology of this paper]:

(1) $\alpha>\mathbf{0}$ and $\gamma=\mathbf{0}$; 
Chang and McAleer, The correct regularity condition and interpretation of asymmetry in EGARCH

$$
\text { (2) } \alpha=\mathbf{0} \text { and } \gamma<\mathbf{0}
$$

Neither of these conditions satisfies the correct condition for leverage, as shown in McAleer and Hafner (2014): (i) under condition (1), if $\gamma=0$, then $\alpha=0$, neither of which is positive, as required; and (ii) under condition (2), if $\alpha=0$, then $\gamma<0$, neither of which is positive, as required.

(3) In discussing “The Exponential GARCH (EGARCH) Model”, the EViews (2017) User's Guide states incorrectly, and without any derivation or explanation, the conditions for leverage and asymmetry as:

"The presence of leverage effects can be tested by the hypothesis that $\gamma<0$.

The impact is asymmetric if $\gamma \neq 0$ ".

It should be clear from the previous discussion that the condition regarding leverage is incorrect as $\alpha$ is omitted and $\gamma$ is not positive, as required. It will be shown below that the condition for asymmetry is also incorrect.

(4) In the RATS (2017) User's Guide, the EGARCH(1,1) model given in equation (8) is discussed under "Asymmetry" in Section 9.3.4 of Chapter 9 (page UG-291), where it is noted that:

"a negative value of $\mathrm{d} 1$ [or $\gamma$ in the terminology of this paper] means that negative residuals tend to produce higher variances in the immediate future.

(This sign convention is used only with the EGARCH)."

From equation (5), it is clear that the parameter $\mathrm{d} 1$ should be positive as it is the variance of a stochastic process. Although the discussion relates to $\mathrm{d} 1$ [or $\gamma$ in the terminology of this paper], no definition of asymmetry is given, so that there is no condition to be examined. Leverage is not discussed.

(5) Engle and Figlewski (2015) also stated the condition for asymmetry incorrectly. Below equation (7) (p. 1000), where the discussion is about $\phi_{3}$ [ or $\gamma$ in the terminology of this paper), the condition for asymmetry is stated incorrectly:

"the parameter $\phi_{3}[=\gamma]$ that measures the asymmetric part of volatility."

The presentation in Engle and Figlewski (2015) should have concentrated on $\phi_{2}$ [or $\alpha$ in the terminology of this paper].

\section{THE CORRECT REGULARITY CONDITION FOR ASYMMETRY IN EGARCH}

In the preceding section, the definitions of leverage and asymmetry were presented in the context of the EGARCH(1,1) model in equation (8). Specifically, the definition of asymmetry is based on the impact of shocks on conditional volatility, which is determined by the means of the derivatives, as will be shown below. This section gives the definition of symmetry, and the regularity condition for asymmetry. In order to obtain the correct regularity condition for asymmetry, the derivatives of $\log h_{t}$ in equation (7) with respect to $\eta_{t-1}$ are given as:

(1) $\alpha+\gamma$ when $\eta_{t-1} \geq 0$

(2) $-\alpha+\gamma$ when $\eta_{t-1}<0$. 
As asymmetry refers to the differential impacts of positive and negative shocks of equal magnitude on volatility, and hence refers to the values of the derivatives in (1) and (2) above, this leads to the following definition of symmetry:

Definition of Symmetry: The impacts of positive and negative shocks of equal magnitude on volatility are the same.

Therefore, symmetry exists for EGARCH if:

$$
\alpha+\gamma=-\alpha+\gamma, \text { that is, } \alpha=0
$$

which leads to the following regularity condition for asymmetry.

Regularity condition for Asymmetry in $\operatorname{EGARCH}(1,1): \alpha \neq 0$.

It follows that the regularity condition for symmetry is $\alpha=0$. As can be seen from the statement in EViews above, and elsewhere, which has been recited and repeated numerous times in the literature, the condition given in the literature for asymmetry concentrates on the incorrect parameter, $\gamma$, rather than the correct parameter, $\alpha$, when asymmetry is defined in terms of the impacts of positive and negative shocks of equal magnitude on volatility.

The following caveat is worth noting for empirical practice.

Caveat: In virtually every empirical example where EGARCH is estimated, the QMLE of $\alpha$ is statistically significant, so $\alpha \neq 0$. Therefore, in practice, EGARCH always displays asymmetry, though not leverage.

\section{CONCLUDING REMARKS}

The paper was concerned with two of the most widely-used univariate conditional volatility models, namely the GARCH model of Engle (1982) and Bollerslev (1986), and the EGARCH model of Nelson (1990, 1991). The EGARCH model is important in capturing asymmetry, which is the different impacts on conditional volatility of positive and negative shocks of equal magnitude, and possibly also leverage, which is the negative correlation between returns shocks and subsequent shocks to volatility. However, Nelson did not provide the regularity conditions for either asymmetry or leverage.

As the condition for asymmetry in EGARCH seems to have been ignored in the literature, or has concentrated on the incorrect condition, without explanation, and hence with associated misleading interpretations, the purpose of the paper was to derive the regularity condition for asymmetry in EGARCH, and to provide the correct interpretation.

When asymmetry is defined in terms of the impacts of positive and negative shocks of equal magnitude on volatility, the condition given in the literature for asymmetry in EGARCH concentrates on the incorrect parameter, $\gamma$, rather than on the correct parameter, $\alpha$. In virtually every empirical example where EGARCH is estimated, the quasi maximum likelihood estimate of $\alpha$ is statistically significant. Therefore, in practice, EGARCH always displays asymmetry. However, EGARCH does not permit leverage, owing to the definition of the parameters in the underlying stochastic process that leads to the EGARCH specification.

This is reminiscent of Indiana Jones who, in Raiders of the Lost Ark, exclaimed that the Nazis were "digging in the wrong place" in searching for the lost Ark of the Covenant. 


\section{ACKNOWLEDGMENTS}

For financial support, the first author wishes to thank the National Science Council, Ministry of Science and Technology (MOST), Taiwan, and the second author is grateful to the National Science Council, Ministry of Science and Technology (MOST), Taiwan and the Australian Research Council.

\section{REFERENCES}

Black, F. (1976). Studies of stock market volatility changes, in Proceedings of the American Statistical Association, Business and Economic Statistics Section, Washington, DC, USA, pp. 177-181.

Bollerslev, T. (1986). Generalised autoregressive conditional heteroscedasticity, Journal of Econometrics, 31, 307-327.

Engle, R. and S. Figlewski (2015). Modeling the dynamics of correlations among implied volatilities, Review of Finance, 19, 991-1018.

Engle, R.F. (1982). Autoregressive conditional heteroscedasticity with estimates of the variance of United Kingdom inflation, Econometrica, 50, 987-1007.

EViews 10 User's Guide, Advanced Single Equation Analysis (2017). Version 10, HIS Global Inc., Irvine, CA, USA.

Glosten, L.R., R. Jagannathan, and D.E. Runkle (1992). On the relation between the expected value and volatility of nominal excess return on stocks, Journal of Finance, 46, 1779-1801.

Ling, S. and M. McAleer (2003). Asymptotic theory for a vector ARMA-GARCH model, Econometric Theory, 19, 278-308.

Martinet, G., and M. McAleer (2018). On the invertibility of $\operatorname{EGARCH}(p, q)$, to appear in Econometric Reviews. McAleer, M. (2014). Asymmetry and leverage in conditional volatility models, Econometrics, 2(3), 145-150.

McAleer, M., and C. Hafner (2014). A one line derivation of EGARCH, Econometrics, 2(2), 92-97.

Nelson, D.B. (1990). ARCH models as diffusion approximations, Journal of Econometrics, 45, 7-38.

Nelson, D.B. (1991). Conditional heteroskedasticity in asset returns: A new approach, Econometrica, 59, 347370 .

RATS 9.0 User's Guide (2017). Version 9.0, Estima Inc., Evanston, IL, USA.

Tsay, R.S. (1987). Conditional heteroscedastic time series models, Journal of the American Statistical Association, 82, 590-604. 